\title{
Crosstalk and Barriers Between the Electron Carriers of the Endoplasmic Reticulum
}

\author{
Gábor Bánhegyi,, Éva Margittai, ${ }^{1}$ András Szarka, ${ }^{2}$ József Mandl, ${ }^{1,3}$ and Miklós Csala ${ }^{1}$
}

\begin{abstract}
Significance: The lumen of the endoplasmic reticulum (ER) constitutes a separate compartment with a special proteome and metabolome. The characteristic redox environment required for the optimal functioning of local pathways is defined by the redox couples of the main electron carriers. These molecules, glutathione, pyridine nucleotides, and ascorbic acid, are present within the ER, but their composition, concentration, and redox state are characteristically different from those observed in other subcellular compartments. Spatial and kinetic barriers contribute to the generation and maintenance of this special redox environment. Recent Advances: The ER redox has usually been considered from the perspective of oxidative protein folding, one of the major functions of the ER. Thus, the lumen has been described as a relatively oxidizing subcellular compartment. Critical Issues: The ER redoxome has been scantily mapped. However, recent observations suggest that the redox systems in reduced and oxidized states are present simultaneously. The concerted actions of transmembrane uptake processes and local oxidoreductases as well as the absence of specific transport and enzyme activities maintain the oxidized state of the thiol-disulfide systems and the reduced state of the pyridine nucleotide redox systems. These states are prerequisites for the normal redox reactions localized in the ER. Future Directions: An outline of the interactions between the major electron carriers of the ER will contribute to a better understanding of human diseases related to ER redox homeostasis.
\end{abstract}

\section{Introduction}

G ROUP TRANSFER using activated carriers is a common mechanism for coupling exergonic and endergonic reactions in metabolism. Electrons carried by a different set of molecules are considered to be the smallest group transferred in redox reactions. In aerobic organisms, the electron carriers transfer electrons from nutrient molecules to the ultimate electron acceptor, molecular oxygen. However, most electron carriers do not deliver electrons directly to oxygen. Instead, the carriers form an electron transfer chain between reduced metabolic intermediates and oxygen that is ranked in order of increasing redox potential. Examples of these systems include the mitochondrial respiratory chain and the cytochrome P450linked electron transfer chain in the ER, both primarily composed of membrane-bound electron carriers. Water-soluble electron carriers are also arranged into chains. Although the Halliwell-Asada pathway (Fig. 1) was originally described in chloroplasts, this pathway is also operative in most organelles found in plant and animal cells. The constituents of this pathway (pyridine nucleotides, ascorbate, and glutathione) are present in cells at high (approximately millimolar) concentrations. Their redox state mirrors the redox environment of a given organelle, and due to their abundance, these constituents can also act as redox buffers.

In addition to group transfer reactions, compartmentation is also a fundamental phenomenon in cell biology. Organelles in the eukaryotic cell and other smaller structures, such as transport vesicles, form cellular compartments that are separated from the cytosol by a phospholipid membrane. Compartmentation enables the cell to separate simultaneously operating metabolic pathways and enhance the efficacy of metabolic activities. Compartments can be characterized by their specific proteomes and metabolomes; the local $\mathrm{pH}$, ionic milieu, and redox conditions can also differ between compartments. A compartment-specific microenvironment is usually required for the optimal functioning of local pathways.

The redox environment of a compartment can be characterized by the composition, concentration, and redox state of its electron carriers. Multiple factors determine the concentration and redox state of an electron carrier in a given

\footnotetext{
${ }^{1}$ Department of Medical Chemistry, Molecular Biology and Pathobiochemistry, Semmelweis University, Budapest, Hungary.

${ }^{2}$ Department of Applied Biotechnology and Food Science, Laboratory of Biochemistry and Molecular Biology, Budapest University of Technology and Economics, Budapest, Hungary.

${ }^{3}$ Pathobiochemistry Research Group of Hungarian Academy of Sciences and Semmelweis University, Budapest, Hungary.
} 


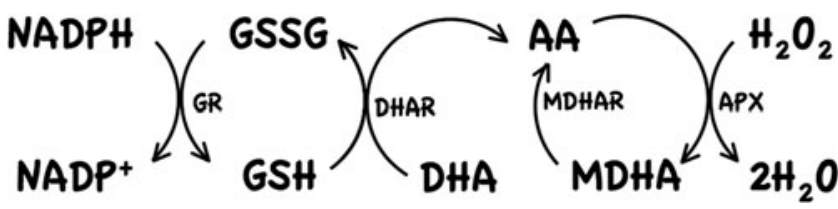

FIG. 1. The electron transfer chain of water-soluble electron carriers. The redox cycling of antioxidants was originally described in the chloroplast and referred to as the Halliwell-Asada pathway or ascorbate-glutathione cycle. AA, ascorbic acid; APX, ascorbate-peroxidase; MDHA, monodehydroascorbate; MDHAR, monodehydroascorbate reductase; DHA, dehydroascorbate; DHAR, dehydroascorbate reductase; GR, glutathione reductase.

compartment (Fig. 2). The carrier molecules are usually synthesized in only one or two compartments: glutathione in the cytosol (30), electron-carrying nucleotides in the cytosol and the mitochondrial matrix $(9,43)$, or ascorbate in the ER (6). The concentration of a carrier molecule depends mainly on its synthesis in its parent compartment(s), whereas its concentration is regulated primarily by membrane transport in the other subcellular compartments. Degradation and further metabolism of a carrier molecule can also modify the local concentration. The redox state of the electron carrier in a given compartment is basically defined by the local oxidoreductases that use the carrier as a cofactor or coenzyme. Connections or disconnections in the electron transfer chain toward oxygen also influence the redox state of each carrier. Finally, selective membrane transport (uptake or release) of the reduced or oxidized form of the carrier can also greatly affect the redox state (Fig. 2).

The lumen of the ER contains important electron carriers. A comprehensive review on the role of electron carriers in ERrelated functions and cellular pathophysiology has been

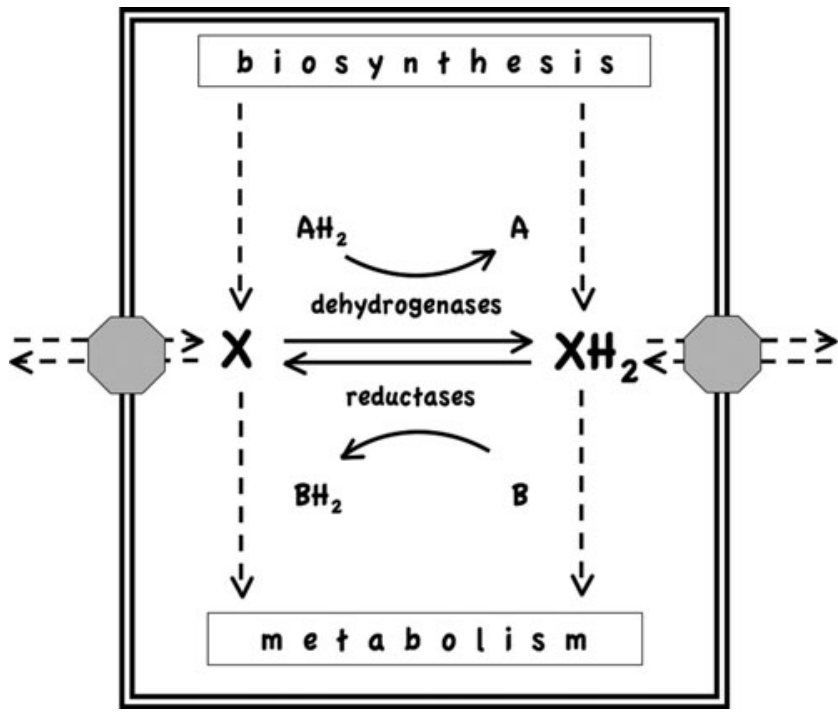

FIG. 2. Factors determining the concentration and redox state of an electron carrier in a subcellular compartment. $X$ and $\mathrm{XH}_{2}$ represent the oxidized and reduced states of the electron carrier, respectively. A and B are substrates for luminal oxidoreductases; octagonal symbols stand for membrane transporters. published recently (13). The aim of the present review is to outline the connections and disconnections (spatial or kinetic) between the three major electron carriers of the ER: glutathione, pyridine nucleotides, and ascorbic acid.

\section{Glutathione, the Main Redox Buffer of the ER Lumen}

Disulfide bond formation in secretory and membrane proteins during oxidative protein folding is one of the most important functions of the ER. This process is catalyzed by a proteinaceous electron relay system in which oxidoreductases, protein disulfide isomerase (PDI), and ER oxidoreductin 1 (Ero1) isozymes are the key players, and oxygen is the ultimate electron acceptor (for a recent review see ref. 16; Fig. 3). Thus, the redox conditions of the thiol-disulfide redox system are considered to be a substantial factor of homeostasis in the ER lumen and have been the focus of much investigation. The potential interference of nonprotein thiols/ disulfides with the oxidation and reduction of protein thiols in the ER lumen underscores the importance of the tight regulation of the ER redox system because changes in either direction would drastically interfere with ER functions.

Similar to other cellular compartments, the major redox buffer of the ER lumen is the glutathione disulfide (GSSG)/ glutathione (GSH) couple. Although total glutathione concentration in the ER lumen and cytosol is presumably alike (in the millimolar range), the state of this redox system is strikingly different when comparing the two sides of the ER membrane. The ratio of [GSSG] to [GSH] is approximately 1:1-1:3 in the ER and the whole secretory pathway (22),

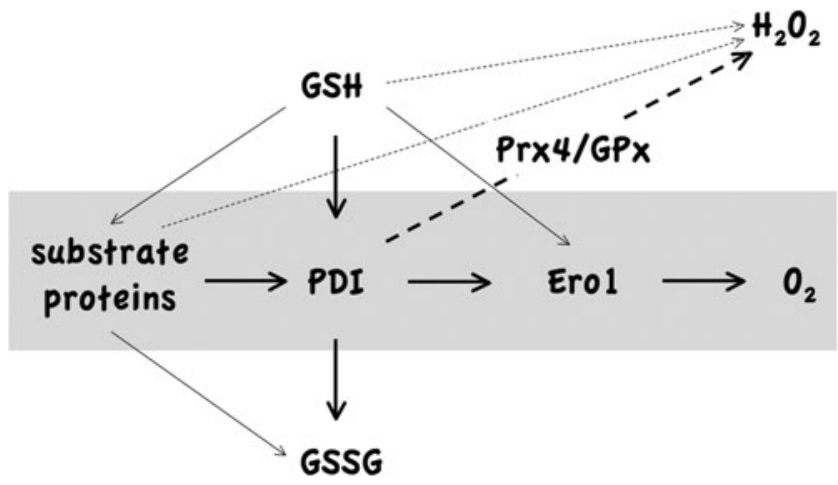

FIG. 3. Redox connections between glutathione and protein thiols within the ER. A proteinaceous electron transfer chain carries electrons from substrate proteins to molecular oxygen exidative protein folding pathway (gray background). Reduced or oxidized glutathione is an alternative electron donor/acceptor in the process, acting mainly via PDI. GSH appears to be necessary for disulfide isomerization by reducing the disulfides of PDI to thiols. This reaction maintains the oxidized state of ER luminal glutathione by generating GSSG, an oxidant for PDI. $\mathrm{H}_{2} \mathrm{O}_{2}$ (produced during oxidative folding or by other oxidases) can oxidize thiols at several points, either mediated by enzymes (PRX4 and GPx) or nonenzymatically (dashed lines). Thick lines represent pathways demonstrated in vivo; thin lines stand for minor or hypothetical reactions or pathways observed in vitro only. ER, endoplasmic reticulum; PRX4, peroxiredoxin 4; GPx, glutathione peroxidase (PDI peroxidase); GSH, glutathione (reduced form); GSSG, glutathione disulfide (oxidized form); PDI, protein disulfide isomerase. 
whereas the cytosolic ratio is approximately 3000-fold lower (21). These ratios show the very efficient compartmentation of GSSG in the ER. Consequently, the ER lumen can be regarded as an oxidative environment, or more exactly, an environment that is more oxidizing than the cytosol.

Because glutathione synthesis has not been reported in the ER, the luminal glutathione pool must be derived from the cytosol. The preferential uptake of GSSG was suggested to be responsible for the peculiar intraluminal milieu and to provide the oxidizing power for disulfide bond formation in secretory proteins (22). However, as later demonstrated, GSH rather than GSSG can cross the ER membrane, suggesting that intraluminal GSSG accumulation is due to GSSG formation by local oxidative processes and the slow release of the compound (7). Thus, glutathione shares the fate of the substrate proteins of oxidative folding: these proteins also enter the ER lumen with cysteine thiols and exit with disulfides. As originally proposed (14), glutathione and protein thiols compete for oxidizing power during disulfide bond formation. $\mathrm{Nu}$ merous connections exist between glutathione and the protein thiols/disulfides of substrate proteins and oxidoreductases of oxidative folding based on the thiol-disulfide exchange (Fig. 3). However, uncontrolled redox reactions between glutathione and the substrate proteins of oxidative folding are inconsistent with effective disulfide bond generation and prevented by steric and kinetic barriers.

The ER membrane represents the most important barrier between GSH and the thiols/disulfides of ER luminal proteins. Accumulation of luminal GSSG and the facilitated, bidirectional diffusion of GSH (7) clearly show that the ER membrane constitutes a selective checkpoint, even for small molecules. Although the molecular background of GSH transport is unknown, its regulation seems to be an important factor in the regulation of ER redox homeostasis. Several observations indicate increased GSH permeability during ER stress due to various mechanisms $(35,38)$. The components of the oxidative folding pathway become hyperoxidized in the absence of cytosolic GSH in plasma membrane-permeabilized cells, whereas the addition of GSH restores normal disulfide formation by allowing disulfide isomerization (39). Thus, the presence of the cytosolic GSH pool and the transport of GSH are indispensable for proper oxidative folding. In agreement with this finding, the removal of the agent causing oxidative stress resulted in the immediate reduction of ER oxidoreductases, for example, ERp57. The reductive force was derived from the cytosol in the form of GSH (23). A shift in the luminal redox potential toward reduction was reported in cells subjected to conditions of ER stress, including experimental and physiological stressors, using fluorescent protein reporters in yeast (38).

In addition to GSH, GSSG can also engage in thiol-disulfide exchange reactions with substrate proteins in the ER, although these reactions are kinetically unfavorable. In vitro experiments showed that supplementation with an adjusted glutathione redox buffer facilitated the refolding of disulfidebonded proteins, even in the absence of oxidoreductases (24). However, the rate of the nonenzymatic reaction was low, and dramatically increased in the presence of CXXC motifcontaining oxidoreductases, such as PDI (57). Thus, the contribution of the nonenzymatic reactions to the total performance of oxidative folding is presumably negligible in a compartment that is well equipped with the proper oxidoreductases.
The rate of GSH oxidation was shown to correlate with Ero1p activity in yeast: regeneration of GSSG was hindered by Ero1p deficiency but enhanced by Ero1p overexpression after dithiothreitol treatment compared with wild-type cells (14). However, in vitro experiments suggest that GSH is a poor substrate for Ero1 (54). These results suggest an indirect link between GSH oxidation and Ero1 activity that can reflect a pathway via PDI or reactive oxygen species (ROS).

Indeed, recent observations show that PDI is the central player not only in the electron relay system of oxidative folding but also in the oxidation/reduction of the luminal glutathione pool (Fig. 3). GSSG-dependent PDI oxidation has been demonstrated in Ero1-deficient cells as a possible alternative pathway for disulfide bond generation (2). In vitro observations confirmed these results, showing a GSSG-dependent oxidation of human PDI and yeast Pdi1p (26). PDI also plays a central role in oxidative folding operated by ROS. Hydrogen peroxide $\left(\mathrm{H}_{2} \mathrm{O}_{2}\right)$, generated as a byproduct of Ero1 or other hypothetical ER flavoproteins, is able to oxidize PDI through peroxiredoxin 4 or PDI peroxidases glutathione peroxidase 7 and $8(59,53,42)$. Elevated levels of high-molecular-weight IgM polymers were observed in peroxiredoxin 4-deficient cells; these levels were attributed to the $\mathrm{H}_{2} \mathrm{O}_{2}$-dependent oxidation of abundant substrate proteins (10). $\mathrm{H}_{2} \mathrm{O}_{2}$ can also sustain native disulfide formation in the absence of peroxidases, as demonstrated in vitro using bovine pancreatic trypsin inhibitor as a model substrate (24).

These recent findings strongly suggest that the high [GSSG]/[GSH] ratio in the ER is due to local oxidase activities involved in protein thiol oxidation; the oxidative luminal environment is the consequence of oxidative protein folding. However, effective alternative pathways help canalize the oxidizing power of $\mathrm{H}_{2} \mathrm{O}_{2}$ and GSSG into oxidative folding. Spatial and kinetic barriers hamper the capacity of GSSG to use its oxidizing potential beyond oxidative folding. Hence, the connection (or disconnection) of the GSH/GSSG redox couple with other luminal electron carriers represents an important aspect of ER redox homeostasis.

\section{Pyridine Nucleotides}

$\mathrm{NAD}(\mathrm{H})$ and its phosphorylated derivative, $\mathrm{NADP}(\mathrm{H})$, are the major water-soluble electron carriers in metabolism (see ref. 46 and references therein). These pyridine nucleotides are presumably present in all subcellular compartments of the eukaryotic cell. The steps of pyridine nucleotide biosynthesis are localized in the cytosol and the mitochondria (43); the origin of the pyridine nucleotide pool in other compartments, such as the peroxisomes and secretory pathway, is enigmatic. $\mathrm{NAD}^{+}$is loaded with electrons by many dehydrogenases participating in the central catabolic pathways of carbohydrate and lipid metabolism, whereas only a few dehydrogenases (glucose-6-phosphate dehydrogenase, 6phosphogluconate dehydrogenase, malic enzyme, and cytosolic isocitrate dehydrogenase) use $\mathrm{NADP}^{+}$specifically as an electron acceptor in the cytosol. NADH principally delivers electrons to the mitochondrial respiratory chain, whereas NADPH provides reducing power for biosynthesis, biotransformation, and antioxidant defense.

The total cellular concentration of pyridine nucleotides is in the submillimolar range; the majority of the pool is present in protein-bound form. The intracellular concentration of 
$\mathrm{NAD}^{+}$plus $\mathrm{NADH}$ is higher than that of $\mathrm{NADP}^{+}$plus NADPH. Moreover, the cytosolic redox state is also different: the $\mathrm{NADP}^{+} / \mathrm{NADPH}$ pool is predominantly in the reduced state, which guarantees the constant maintenance of the antioxidant milieu. In contrast, the cytosolic (and mitochondrial) $\mathrm{NAD}^{+} / \mathrm{NADH}$ system is overwhelmingly present in the oxidized state.

The lumen of the ER contains pyridine nucleotidedependent enzymes (5), hexose-6-phosphate dehydrogenase (H6PD) and 11 -hydroxysteroid dehydrogenase type 1 $(11 \beta$ HSD1). However, data are sparse on the concentration and composition of the ER luminal pyridine nucleotide pool. Because tools are not available for the separate detection of $\mathrm{NAD}^{+} / \mathrm{NADH}$ or $\mathrm{NADP}^{+} / \mathrm{NADPH}$ in vivo or in cellular systems, the present data have been collected from microsomal fractions using enzymatic methods. Unfortunately, an unequivocal conclusion cannot be drawn from these conflicting results with respect to the composition, the concentration, or the redox state of the luminal pyridine nucleotides $(11,25)$.

However, the redox conditions of the pyridine nucleotide pool of the ER can be studied indirectly through the activity of the prereceptorial glucocorticoid activating machinery. The intraluminal oxidoreductase $11 \beta \mathrm{HSD} 1$ activates glucocorticoid precursors by reduction, and its activity is a major determinant of the local glucocorticoid hormone levels (17) The reaction catalyzed by $11 \beta \mathrm{HSD} 1$ is reversible; the actual direction is largely dependent on the redox state of the $\mathrm{NADPH} / \mathrm{NADP}^{+}$system (18). In fact, the physiological direction of the process is the strongest indirect evidence for a high ER luminal [NADPH]/[NADP ${ }^{+}$] ratio, which has not yet been directly determined.

The high ER luminal [NADPH]/[NADP $\left.{ }^{+}\right]$ratio is generated and maintained by luminal $\mathrm{NADP}^{+}$-dependent dehydrogenases. H6PD is the most important NADPH-generating enzyme in the lumen and has been shown to have a tight functional $(3,5,28)$ and physical $(4)$ connection with $11 \beta \mathrm{HSD} 1$

F4 (Fig. 4). The low $K_{M}$ of the enzyme toward glucose-6phosphate ensures that H6PD is active at low substrate concentrations and in the presence of the competing luminal glucose-6-phosphatase, which is a high-capacity but lowaffinity enzyme (55). The absence of NADPH-dependent feedback inhibition guarantees that H6PD can generate a very high $[\mathrm{NADPH}] /\left[\mathrm{NADP}^{+}\right]$ratio (50 and references therein). The role of other $\mathrm{NADP}^{+}$-dependent dehydrogenases present in the lumen, such as isocitrate dehydrogenase (33) and malic enzyme (56), in redox homeostasis of the ER lumen has not been clarified. In addition to $11 \beta \mathrm{HSD} 1$, other NADPHconsuming enzymes may be present in the ER lumen, such as Ncb5or [NAD(P)H cytochrome b5 oxidoreductase], that presumably transfer electrons from NADPH to the $\Delta 9$ fatty acid desaturase system (27) and ER flavoprotein associated with degradation, a putative component of ER-associated degradation (ERAD) (48). However, the luminal presence of Ncb5or has not been proven unequivocally. Moreover, some members of the short-chain dehydrogenase/reductase family and certain cytochrome P450 isozymes have a luminal orientation, suggesting that these enzymes might utilize luminal NADPH (Fig. 4). Other enzymes embedded in the ER membrane (NADPH oxidase 4, most cytochrome P450 isozymes) that use cytosolic NADPH as a cofactor may also oxidize luminal $\mathrm{NADPH}$ via ROS generation. Indeed, $\mathrm{H}_{2} \mathrm{O}_{2}$ is able to deplete

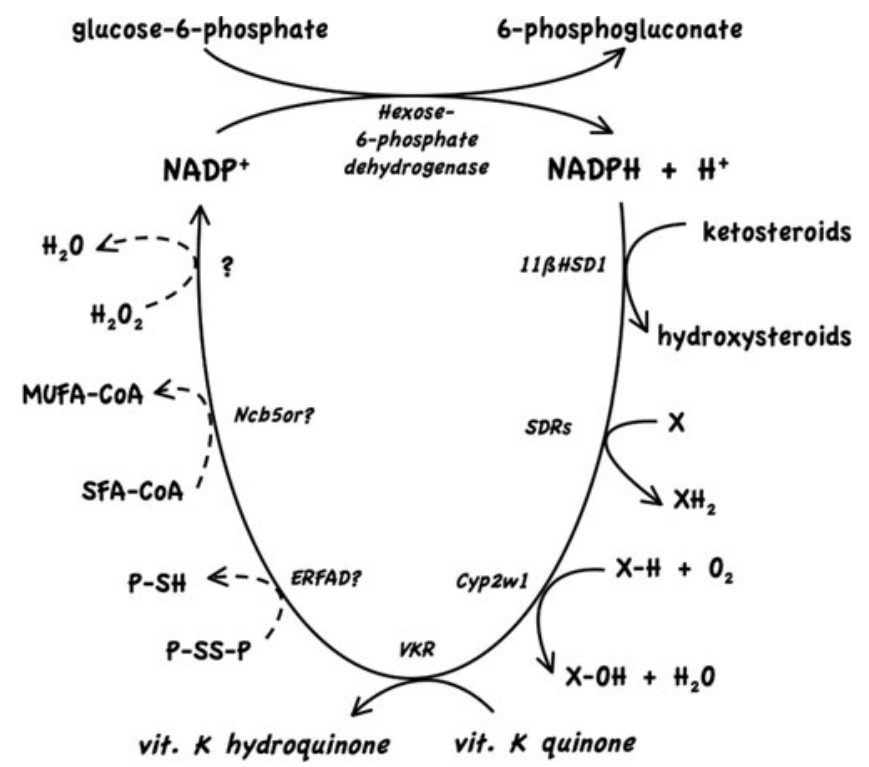

FIG. 4. Redox connections of pyridine nucleotides within the ER. The redox status of the luminal $\mathrm{NADP}^{+} / \mathrm{NADPH}$ system is influenced by a major dehydrogenase and several reductive reactions competing for NADPH. Dashed lines represent the hypothetical NADPH-dependent reactions. $11 \beta$ HSD1, 11 $\beta$-hydroxysteroid dehydrogenase type 1; MUFA, monounsaturated fatty acid; P-SH, protein thiol; P-SS-P, protein disulfide; SDR, short-chain dehydrogenase/reductase; SFA, saturated fatty acid; VKR, vitamin $\mathrm{K}$ reductase (NADPH dependent); vit, vitamin; ERFAD, endoplasmic reticulum flavoprotein associated with degradation.

ER NADPH in vivo and in vitro (Margittai et al., unpublished observation).

Because the ER membrane is hardly permeable to pyridine nucleotides (45), luminal oxidoreductase enzymes must use an $\mathrm{NADP}(\mathrm{H})$ pool that is functionally separate from the cytosolic pool. The NADPH/NADP ${ }^{+}$redox pair is tightly coupled to the GSH/GSSG and ascorbate/dehydroascorbate (DHA) systems in the cytosol. GSSG is mainly reduced by the NADPH-dependent enzyme glutathione reductase (GR), whereas DHA can be reduced by NADPH- (15) or GSHdependent (31) reductases. NADPH-dependent DHA reductase has not been observed in the ER, and GR has been absent from rat liver microsomes (45), suggesting that the pyridine nucleotide redox system is separated from the GSH/GSSG couple in the ER lumen. The separation of the two redox systems is further supported by the following observations: the addition of GSH or GSSG does not influence the redox state of pyridine nucleotides in liver microsomes (45), and reduced or oxidized pyridine nucleotides are unable to affect the redox state of microsomal thiols (45) or influence oxidative protein folding (36).

Nevertheless, some observations suggest that indirect connections might exist between the GSH/GSSG and the $\mathrm{NADPH} / \mathrm{NADP}^{+}$systems in the ER. Deletion of H6PD activates the unfolded protein response pathway and induces skeletal myopathy in mice (29). Silencing of the enzyme provokes ER stress-dependent autophagy in HepG2 cells (51). These findings may indicate the perturbation of oxidative folding, which is the prototypic cause of ER stress and the unfolded protein response. However, recent studies clearly 
show that altered lipid metabolism in obesity can also cause ER stress in the liver by disrupting calcium homeostasis (19). Because pyridine nucleotides play an essential role in the metabolism of lipids, NADPH depletion might also lead to ER stress without affecting oxidative folding.

Other possible connections between the two redox systems include the competition between NADPH and thiols for $\mathrm{H}_{2} \mathrm{O}_{2}$ detoxification and the putative involvement of NADPH in disulfide bond reduction during the ERAD; however, these possibilities lack experimental verification.

\section{Ascorbate and Dehydroascorbic Acid}

Among water-soluble electron carriers, ascorbate has the most intimate relationship with the ER; the last step of its synthesis is catalyzed by the ER membrane-bound flavoprotein gulonolactone oxidase (GLO) in the hepatocytes of most animals (see ref. 6 and references therein). Interestingly, in humans and a few animal species, GLO is inactive due to a mutation in its active site; thus, these species need to ingest vitamin $C$ (ascorbate) in their diet. Prediction of membrane topology and indirect experimental evidence show that the active site of GLO is located in the ER lumen; consequently, de novo synthesized ascorbate first appears in the lumen of the hepatic ER, which presumably results in high local ascorbate concentration. Moreover, $\mathrm{H}_{2} \mathrm{O}_{2}$, the byproduct of the reaction, is also produced in the lumen, contributing to the oxidation of GSH and, consequently, the oxidative character of the compartment (47). Although ascorbate synthesis is limited to the ER of hepatocytes, ascorbate can enter the ER lumen in other cells as well. Microsomal transport activity specific to oxidized ascorbate (dehydroascorbic acid) has been described (8); this transporter can ensure dehydroascorbic acid (and consequently ascorbate) supply for the ER lumen in cells devoid of GLO. This transporter has not been identified at the molecular level; its features suggest that it belongs to the family of GLUT transporters. However, data are not available on the luminal concentration and redox state of ascorbate in hepatocytes or other cells not expressing GLO. The rapid, spontaneous decomposition of dehydroascorbic acid at a neutral $\mathrm{pH}$ suggests that the concentration of ascorbate significantly surpasses that of dehydroascorbic acid.

Ascorbate and dehydroascorbic acid, an excellent electron donor and acceptor, respectively, maintain vital functions in the ER lumen. Ascorbate-utilizing reactions, such as the detoxification of ROS and redox reactions using ascorbate as a cofactor, take advantage of the susceptibility of ascorbate to oxidation. These reactions yield semidehydroascorbic acid radical and/or dehydroascorbic acid (Fig. 1). Ascorbate acts as an antioxidant in the lumen, similar to other compartments of the cell. Moreover, ascorbate is a cofactor for hydroxylases (prolyl- and lysyl-hydroxylases; ref. 40) participating in the posttranslational modification of extracellular matrix proteins.

A close and unidirectional connection exists between the ascorbate/DHA and thiol/disulfide redox systems (Fig. 5). Whereas dehydroascorbic acid is known as a thiol oxidant, GSSG does not oxidize ascorbate. Dehydroascorbic acid can accept electrons from various components of the oxidative folding system, such as glutathione (37), substrate proteins (49), and PDI $(41,58)$, via nonenzymatic reactions. These reactions suggest that dehydroascorbic acid might be involved in the electron transfer chain responsible for oxidative protein folding.

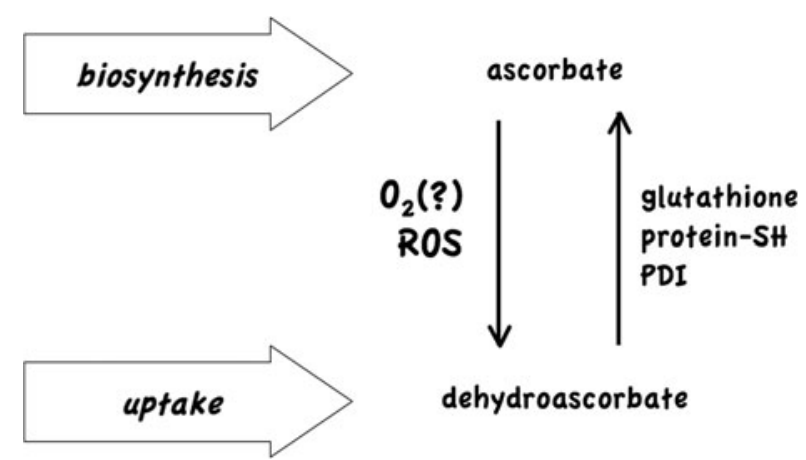

FIG. 5. Redox connections of ascorbate within the ER. The ascorbate/dehydroascorbate redox couple is able to promote continuous thiol-oxidizing machinery. Dehydroascorbate oxidizes GSH and protein thiols. Ascorbate produced in these reactions can be reoxidized by ROS or local ascorbate oxidase activity. The cycle can be replenished by dehydroascorbate uptake, or by de novo synthesis in hepatocytes. ROS, reactive oxygen species.

The oxidant behavior of dehydroascorbic acid, together with its uptake into the ER lumen and the presence of microsomal ascorbate oxidase activity (52), suggests a model of an alternative electron transfer chain that contributes to oxidative protein folding (Fig. 5). The addition of gulonolactone, ascorbate, or dehydroascorbic acid was shown to enhance oxidation of protein thiols in rat liver microsomes (12). The physiological role of ascorbate in oxidative protein folding was supported by the observation that ascorbate deficiency (scurvy) causes ER stress and apoptosis in the liver of guinea pigs (32). ER stress was elicited as a consequence of defective dehydroascorbic acid-mediated oxidation rather than the absence of the antioxidant ascorbate. Nevertheless, the indirect effects of scurvy on ER function or defects in collagen hydroxylation contributing to the development of ER stress cannot be ruled out. The relative contribution of the ascorbate/DHA redox system to oxidative folding cannot be estimated based on available experimental evidence.

Only sparse data are available on the connection of the ascorbate/dehydroascorbic acid redox couple with other electron carriers of the ER. NADPH-dependent dehydroascorbic acid reductases that are present in the cytosol (15) have not been reported in the ER.

\section{Concluding Remarks}

The special functions of the luminal compartment of the ER are supported by peculiar redox conditions. The membranous barrier between the cytosol and the lumen allows the maintenance of separate redox environments. Whereas the luminal pyridine nucleotide pool appears to be completely isolated, the reduced or oxidized form of other electron carriers, GSH and dehydroascorbic acid, can be transported by bidirectional facilitated diffusion. Both uptake processes can contribute to the functioning of oxidative protein folding; GSH allows isomerization of disulfide bonds, whereas dehydroascorbic acid acts as a thiol oxidant.

In addition to compartmentation, functional barriers also limit crosstalk between the redox systems in the ER. Whereas in other compartments the carriers form a continuous electron transfer chain, the luminal chain in the ER is broken due to the 


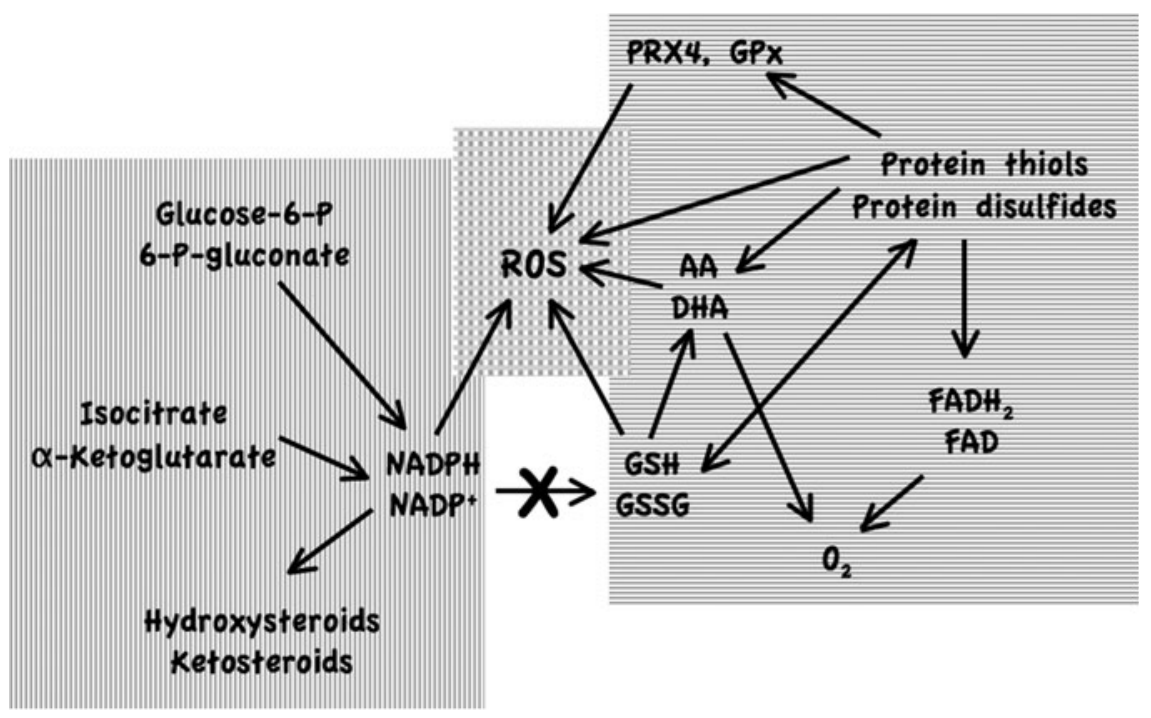

FIG. 6. Crosstalk and barriers between the redox systems in the ER. Connections between luminal electron carriers are shown. Arrows indicate the direction of electron transfer in each relationship. Redox-active compounds of the lumen form a bicentral network around PDI and pyridine nucleotides due to uncoupling between the thiol/disulfide and NADPH/ $\mathrm{NADP}^{+}$systems. The two systems, however, communicate through competition for ROS (mainly for $\mathrm{H}_{2} \mathrm{O}_{2}$ ) with multiple, efficient enzymatic and nonenzymatic reactions, ensuring the priority of thiol oxidation in the competition. missing GR. This uncoupling preserves the biochemical basis of two vital processes in the ER, oxidative protein folding and (reductive) prereceptorial glucocorticoid activation, by allowing the peaceful coexistence of redox couples in antagonistic states. Due to the uncoupling between the two systems (Fig. 6), the redox-active compounds of the lumen form a bicentral network around PDI and pyridine nucleotides.

The third center of the ER redox network is composed of ROS (Fig. 6). Detoxification of ROS is a common task of the two separate redox systems. The abundance of potential ROSgenerating enzymes and poor representation of antioxidant enzymes in the ER can lead to the accumulation of oxidative power in the lumen, which can be directed toward oxidative folding via multiple mechanisms in a stochastic way (34). Peroxiredoxin 4, PDI peroxidases, and the ascorbate-DHA redox couple can effectively recycle the oxidizing power of $\mathrm{H}_{2} \mathrm{O}_{2}$ into oxidative folding via PDI family members. Eventually, cysteinyl thiols of the newly synthesized luminal proteins supply the reducing power for the antioxidant defense of the compartment. The multiplicity, redundancy, and interchangeability of oxidizing agents ensure that oxidative folding can continue without fatal disturbances even in the absence of certain oxidants.

Whereas membranous and functional barriers are crucial to separate and maintain the special luminal redox environment, close interactions with other cellular compartments could allow the exchange of redox-active components. From this perspective, the recently described functional/morphological contact site between the ER and the mitochondria, the mitochondria-associated membrane (MAM), could have an essential impact on ER redox. This specialized membrane domain is enriched in transporters and channels involved in calcium signaling (44) and has several components of the oxidative folding machinery. Ero1 $\alpha$ was located in the MAM and showed a redox-dependent migration between the ER and the MAM fraction (20). The vicinity of Ero1 $\alpha$ to mitochondria is reasonable in terms of its regulatory effects on $\mathrm{Ca}^{2+}$ homeostasis (1) and its need for an FAD prosthetic group synthesized in the mitochondria (9). These connections can also facilitate the transfer of redox-active compounds (e.g., FAD and ROS) between the two organelles.
Our knowledge of ER redox homeostasis has been restricted to the thiol-disulfide redox system and its relation to oxidative folding. The appropriate tools needed to investigate the other redox systems of the ER are missing. The development of ER-targeted redox sensors specific to pyridine nucleotides, ascorbate, or ROS is indispensable for mapping the ER redoxome and understanding the redox connections and their role in the regulation of ER-resident pathways. The elucidation of redox-driven ER stress mechanisms, involving or omitting the unfolded protein response, will provide further insight into the pathomechanism of ER stress-related diseases.

\section{Acknowledgments}

This work was supported by the Hungarian Scientific Research Fund (OTKA NN 78300 and 75275); by the János Bolyai Research Scholarship of the Hungarian Academy of Sciences (to M.Cs., A.Sz., and É.M.); by the Ministry of Health, Hungary (ETT 037 and 116/2009); and by the New Széchenyi Plan (Project ID: TÁMOP-4.2.1/B-09/1/KMR-2010-0002 and TÁMOP-4.2.1./B-09/1/KMR-2010-0001). This work is also connected to the scientific program of the "Development of quality-oriented and harmonized $\mathrm{R}+\mathrm{D}+\mathrm{I}$ strategy and functional model at BME" project.

\section{Author Disclosure Statement}

No competing financial interests exist.

\section{References}

1. Anelli T, Bergamelli L, Margittai E, Rimessi A, Fagioli C, Malgaroli A, Pinton P, Ripamonti M, Rizzuto R, and Sitia R. Erol $\alpha$ regulates $\mathrm{Ca}^{2+}$ fluxes at the endoplasmic reticulummitochondria interface (MAM). Antioxid Redox Signal 2011 [Epub ahead of print].

2. Appenzeller-Herzog C, Riemer J, Zito E, Chin KT, Ron D, Spiess M, and Ellgaard L. Disulphide production by Ero1 $\alpha-$ PDI relay is rapid and effectively regulated. EMBO J 29: 3318-3329, 2010.

3. Atanasov AG, Nashev LG, Schweizer RA, Frick C, and Odermatt A. Hexose-6-phosphate dehydrogenase determines 
the reaction direction of $11 \beta$-hydroxysteroid dehydrogenase type 1 as an oxoreductase. FEBS Lett 571: 129-133, 2004.

4. Atanasov AG, Nashev LG, Gelman L, Legeza B, Sack R, Portmann R, and Odermatt A. Direct protein-protein interaction of $11 \beta$-hydroxysteroid dehydrogenase type 1 and hexose-6-phosphate dehydrogenase in the endoplasmic reticulum lumen. Biochim Biophys Acta 1783: 1536-1543, 2008.

5. Bánhegyi G, Benedetti A, Fulceri R, and Senesi S. Cooperativity between $11 \beta$-hydroxysteroid dehydrogenase type 1 and hexose-6-phosphate dehydrogenase in the lumen of the endoplasmic reticulum. J Biol Chem 279: 27017-27021, 2004.

6. Bánhegyi G, Braun L, Csala M, Puskás F, and Mandl J. Ascorbate metabolism and its regulation in animals. Free Radic Biol Med 23: 793-803, 1997.

7. Bánhegyi G, Lusini L, Puskás F, Rossi R, Fulceri R, Braun L, Mile V, di Simplicio P, Mandl J, and Benedetti A. Preferential transport of glutathione versus glutathione disulfide in rat liver microsomal vesicles. J Biol Chem 274: 12213-12216, 1999.

8. Bánhegyi G, Marcolongo P, Puskás F, Fulceri R, Mandl J, and Benedetti A. Dehydroascorbate and ascorbate transport in rat liver microsomal vesicles. J Biol Chem 273: 2758-2762, 1998.

9. Barile M, Brizio C, Valenti D, De Virgilio C, and Passarella S. The riboflavin/FAD cycle in rat liver mitochondria. Eur $J$ Biochem 267: 4888-4900, 2000.

10. Bertolotti M, Yim SH, Garcia-Manteiga JM, Masciarelli S, Kim YJ, Kang MH, Iuchi Y, Fujii J, Vené R, Rubartelli A, Rhee SG, and Sitia R. B- to plasma-cell terminal differentiation entails oxidative stress and profound reshaping of the antioxidant responses. Antioxid Redox Signal 13: 1133-1144, 2010.

11. Bublitz C and Lawler CA. The levels of nicotinamide nucleotides in liver microsomes and their possible significance to the function of hexose phosphate dehydrogenase. Biochem J 245: 263-267, 1987.

12. Csala M, Braun L, Mile V, Kardon T, Szarka A, Kupcsulik P, Mandl J, and Bánhegyi G. Ascorbate-mediated electron transfer in protein thiol oxidation in the endoplasmic reticulum. FEBS Lett 460: 539-543, 1999.

13. Csala M, Margittai É, and Bánhegyi G. Redox control of endoplasmic reticulum function. Antioxid Redox Signal 13: 77-108, 2010.

14. Cuozzo JW and Kaiser CA. Competition between glutathione and protein thiols for disulfide-bond formation. Nat Cell Biol 1: 130-135, 1999.

15. Del Bello B, Maellaro E, Sugherini L, Santucci A, Comporti $\mathrm{M}$, and Casini AF. Purification of NADPH-dependent dehydroascorbate reductase from rat liver and its identification with $3 \alpha$-hydroxysteroid dehydrogenase. Biochem J 304: 385390, 1994

16. Depuydt M, Messens J, and Collet JF. How proteins form disulfide bonds. Antioxid Redox Signal 15: 49-66, 2011.

17. Draper N and Stewart PM. 11 $\beta$-hydroxysteroid dehydrogenase and the pre-receptor regulation of corticosteroid hormone action. J Endocrinol 186: 251-271, 2005.

18. Dzyakanchuk AA, Balázs Z, Nashev LG, Amrein KE, and Odermatt A. 11 $\beta$-hydroxysteroid dehydrogenase 1 reductase activity is dependent on a high ratio of NADPH/NADP ${ }^{+}$ and is stimulated by extracellular glucose. Mol Cell Endocrinol 301: 137-141, 2009.

19. Fu S, Yang L, Li P, Hofmann O, Dicker L, Hide W, Lin X, Watkins SM, Ivanov AR, and Hotamisligil GS. Aberrant lipid metabolism disrupts calcium homeostasis causing liver endoplasmic reticulum stress in obesity. Nature 473: 528-531, 2011.

20. Gilady SY, Bui M, Lynes EM, Benson MD, Watts R, Vance $\mathrm{JE}$, and Simmen T. Erolalpha requires oxidizing and nor- moxic conditions to localize to the mitochondria-associated membrane (MAM). Cell Stress Chaperones 15: 619-629, 2010.

21. Gutscher M, Pauleau AL, Marty L, Brach T, Wabnitz GH, Samstag Y, Meyer AJ, and Dick TP. Real-time imaging of the intracellular glutathione redox potential. Nat Methods 5: 553 $559,2008$.

22. Hwang C, Sinskey AJ, and Lodish HF. Oxidized redox state of glutathione in the endoplasmic reticulum, Science 257: 1496-1502, 1992.

23. Jessop CE and Bulleid NJ. Glutathione directly reduces an oxidoreductase in the endoplasmic reticulum of mammalian cells. J Biol Chem 279: 55341-55347, 2004.

24. Karala AR, Lappi AK, Saaranen MJ, and Ruddock LW. Efficient peroxide-mediated oxidative refolding of a protein at physiological $\mathrm{pH}$ and implications for oxidative folding in the endoplasmic reticulum. Antioxid Redox Signal 11: 963970, 2009.

25. Kereszturi É, Kálmán FS, Kardon T, Csala M, and Bánhegyi G. Decreased prereceptorial glucocorticoid activating capacity in starvation due to an oxidative shift of pyridine nucleotides in the endoplasmic reticulum. FEBS Lett 584: 4703-4708, 2010.

26. Lappi AK and Ruddock LW. Reexamination of the role of interplay between glutathione and protein disulfide isomerase. J Mol Biol 409: 238-249, 2011.

27. Larade K, Jiang Z, Zhang Y, Wang W, Bonner-Weir S, Zhu $\mathrm{H}$, and Bunn HF. Loss of Ncb5or results in impaired fatty acid desaturation, lipoatrophy, and diabetes. J Biol Chem 283: 29285-29291, 2008.

28. Lavery GG, Walker EA, Draper N, Jeyasuria P, Marcos J, Shackleton $\mathrm{CH}$, Parker KL, White PC, and Stewart PM. Hexose-6-phosphate dehydrogenase knock-out mice lack $11 \beta$-hydroxysteroid dehydrogenase type 1-mediated glucocorticoid generation. J Biol Chem 281: 6546-6551, 2006.

29. Lavery GG, Walker EA, Turan N, Rogoff D, Ryder JW, Shelton JM, Richardson JA, Falciani F, White PC, Stewart PM, Parker KL, and McMillan DR. Deletion of hexose-6phosphate dehydrogenase activates the unfolded protein response pathway and induces skeletal myopathy. J Biol Chem 283: 8453-8461, 2008.

30. Lu SC. Regulation of glutathione synthesis. Mol Aspects Med 30: 42-59, 2009.

31. Maellaro E, Del Bello B, Sugherini L, Comporti M, and Casini AF. Purification and characterization of glutathionedependent dehydroascorbate reductase from rat liver. Methods Enzymol 279: 30-35, 1997.

32. Margittai É, Bánhegyi G, Kiss A, Nagy G, Mandl J, Schaff Z, and Csala M. Scurvy leads to endoplasmic reticulum stress and apoptosis in the liver of Guinea pigs. J Nutr 135: 25302534, 2005.

33. Margittai É and Bánhegyi G. Isocitrate dehydrogenase: a NADPH-generating enzyme in the lumen of the endoplasmic reticulum. Arch Biochem Biophys 471: 184-190, 2008.

34. Margittai É and Bánhegyi G. Oxidative folding in the endoplasmic reticulum: towards a multiple oxidant hypothesis? FEBS Lett 584: 2995-2998, 2010.

35. Margittai É, Löw P, Szarka A, Csala M, Benedetti A, and Bánhegyi G. Intraluminal hydrogen peroxide induces a permeability change of the endoplasmic reticulum membrane. FEBS Lett 582: 4131-4136, 2008.

36. Marquardt T, Hebert DN, and Helenius A. Posttranslational folding of influenza hemagglutinin in isolated endoplasmic reticulum-derived microsomes. J Biol Chem 268: 19618$19625,1993$. 
37. May JM, Qu ZC, Whitesell RR, and Cobb CE. Ascorbate recycling in human erythrocytes: role of GSH in reducing dehydroascorbate. Free Radic Biol Med 20: 543-551, 1996.

38. Merksamer PI, Trusina A, and Papa FR. Real-time redox measurements during endoplasmic reticulum stress reveal interlinked protein folding functions. Cell 135: 933-947, 2008.

39. Molteni SN, Fassio A, Ciriolo MR, Filomeni G, Pasqualetto E, Fagioli C, and Sitia R. Glutathione limits Ero1-dependent oxidation in the endoplasmic reticulum. J Biol Chem 279: 32667-32673, 2004.

40. Myllyharju J. Prolyl 4-hydroxylases, key enzymes in the synthesis of collagens and regulation of the response to hypoxia, and their roles as treatment targets. Ann Med 40: 402-417, 2008

41. Nardai G, Braun L, Csala M, Mile V, Csermely P, Benedetti A, Mandl J, and Bánhegyi G. Protein-disulfide isomeraseand protein thiol-dependent dehydroascorbate reduction and ascorbate accumulation in the lumen of the endoplasmic reticulum. J Biol Chem 276: 8825-8828, 2001.

42. Nguyen VD, Saaranen MJ, Karala AR, Lappi AK, Wang L, Raykhel IB, Alanen HI, Salo KE, Wang CC, and Ruddock LW. Two endoplasmic reticulum PDI peroxidases increase the efficiency of the use of peroxide during disulfide bond formation. J Mol Biol 406: 503-515, 2011.

43. Nikiforov A, Dölle C, Niere M, and Ziegler M. Pathways and subcellular compartmentation of NAD biosynthesis in human cells: from entry of extracellular precursors to mitochondrial NAD generation. J Biol Chem 286: 21767-21778, 2011.

44. Patergnani S, Suski JM, Agnoletto C, Bononi A, Bonora M, De Marchi E, Giorgi C, Marchi S, Missiroli S, Poletti F, Rimessi A, Duszynski J, Wieckowski MR, and Pinton P. Calcium signaling around Mitochondria Associated Membranes (MAMs). Cell Commun Signal 9: 19, 2011.

45. Piccirella S, Czegle I, Lizák B, Margittai É, Senesi S, Papp E, Csala M, Fulceri R, Csermely P, Mandl J, Benedetti A, and Bánhegyi G. Uncoupled redox systems in the lumen of the endoplasmic reticulum. Pyridine nucleotides stay reduced in an oxidative environment. J Biol Chem 281: 4671-4677, 2006.

46. Pollak N, Dölle C, and Ziegler M. The power to reduce: pyridine nucleotides-small molecules with a multitude of functions. Biochem J 402: 205-218, 2007.

47. Puskás F, Braun L, Csala M, Kardon T, Marcolongo P, Benedetti A, Mandl J, and Bánhegyi G. Gulonolactone oxidase activity-dependent intravesicular glutathione oxidation in rat liver microsomes. FEBS Lett 430: 293-296, 1998.

48. Riemer J, Appenzeller-Herzog C, Johansson L, Bodenmiller B, Hartmann-Petersen R, and Ellgaard L. A luminal flavoprotein in endoplasmic reticulum associated degradation. Proc Natl Acad Sci U S A 106: 14831-14836, 2009.

49. Saaranen MJ, Karala AR, Lappi AK, and Ruddock LW. The role of dehydroascorbate in disulfide bond formation. Antioxid Redox Signal 12: 15-25, 2010.

50. Senesi S, Csala M, Marcolongo P, Fulceri R, Mandl J, Bánhegyi G, and Benedetti A. Hexose-6-phosphate dehydrogenase in the endoplasmic reticulum. Biol Chem 391: 1-8, 2010

51. Száraz P, Bánhegyi G, and Benedetti A. Altered redox state of luminal pyridine nucleotides facilitates the sensitivity towards oxidative injury and leads to endoplasmic reticulum stress dependent autophagy in HepG2 cells. Int J Biochem Cell Biol 42: 157-166, 2010.

52. Szarka A, Stadler K, Jenei V, Margittai E, Csala M, Jakus J, Mandl J, and Bánhegyi G. Ascorbyl free radical and dehydroascorbate formation in rat liver endoplasmic reticulum. J Bioenerg Biomembr 34: 317-323, 2002.
53. Tavender TJ, Springate JJ, and Bulleid NJ. Recycling of peroxiredoxin IV provides a novel pathway for disulphide formation in the endoplasmic reticulum. EMBO J 29: 41854197, 2010.

54. Tu BP and Weissman JS. The FAD- and $\mathrm{O}_{2}$-dependent reaction cycle of Ero1-mediated oxidative protein folding in the endoplasmic reticulum. Mol Cell 10: 983-994, 2002.

55. van Schaftingen E and Gerin I. The glucose-6-phosphatase system. Biochem J 362: 513-532, 2002.

56. Wang X, Mick GJ, Maser E, and McCormick K. Manifold effects of palmitoylcarnitine on endoplasmic reticulum metabolism: 11 $\beta$-hydroxysteroid dehydrogenase 1, flux through hexose-6-phosphate dehydrogenase and NADPH concentration. Biochem J 437: 109-115, 2011.

57. Weissman JS and Kim PS. Efficient catalysis of disulphide bond rearrangements by protein disulphide isomerase. Nature 365: 185-188, 1993.

58. Wells WW, Xu DP, Yang YF, and Rocque PA. Mammalian thioltransferase (glutaredoxin) and protein disulfide isomerase have dehydroascorbate reductase activity. J Biol Chem 265: 15361-15364, 1990.

59. Zito E, Melo EP, Yang Y, Wahlander Å, Neubert TA, and Ron D. Oxidative protein folding by an endoplasmic reticulum-localized peroxiredoxin. Mol Cell 40: 787-797, 2010.

$$
\begin{array}{r}
\text { Address correspondence to: } \\
\text { Prof. Gábor Bánhegyi } \\
\text { Department of Medical Chemistry } \\
\text { Molecular Biology and Pathobiochemistry } \\
\text { Semmelweis University } \\
\text { POB } 260 \\
\text { Budapest } 1444 \\
\text { Hungary } \\
\text { E-mail: banhegyi@eok.sote.hu }
\end{array}
$$

Date of first submission to ARS Central, November 25, 2011; date of acceptance, December 5, 2011.

$\begin{aligned} & \text { Abbreviations Used } \\ & 11 \beta \mathrm{HSD} 1=11 \beta \text {-hydroxysteroid dehydrogenase type } 1 \\ & \mathrm{AA}=\text { ascorbic acid } \\ & \mathrm{APX}=\text { ascorbate-peroxidase } \\ & \mathrm{DHA}=\text { dehydroascorbate } \\ & \mathrm{DHAR}=\text { dehydroascorbate reductase } \\ & \mathrm{ER}=\text { endoplasmic reticulum } \\ & \mathrm{ERAD}=\text { endoplasmic reticulum-associated } \\ & \mathrm{ERFAD}=\text { endegradation } \\ & \mathrm{Ero} 1=\text { associated with degradation } \\ & \mathrm{GLO}=\text { gulonolactone oxidase } \\ & \mathrm{GPx}=\text { glutathione peroxidase } \\ & \mathrm{GR}=\text { glutathione reductase } \\ & \mathrm{GSH}=\text { glutathione (reduced form) } \\ & \mathrm{GSSG}=\text { glutathione disulfide (oxidized form) } \\ & \mathrm{H}_{2} \mathrm{O}_{2}=\text { hydrogen peroxide } \\ & \mathrm{H} 6 \mathrm{PD}=\text { hexose- } 6 \text {-phosphate dehydrogenase } \\ & \mathrm{MAM}=\text { mitochondria-associated membrane } \\ & \mathrm{MDHA}=\text { monodehydroascorbate } \\ & \mathrm{MDHAR}=\text { monodehydroascorbate reductase } \\ &\end{aligned}$


Abbreviations Used (Cont.)

MUFA $=$ monounsaturated fatty acid

Ncb5or $=$ NAD $(\mathrm{P}) \mathrm{H}$ cytochrome b5 oxidoreductase

$\mathrm{PDI}=$ protein disulfide isomerase

PRX $4=$ peroxiredoxin 4

$\mathrm{P}-\mathrm{SH}=$ protein thiol
$\mathrm{P}-\mathrm{SS}-\mathrm{P}=$ protein disulfide

ROS $=$ reactive oxygen species

$\mathrm{SDR}=$ short-chain dehydrogenase/reductase

SFA $=$ saturated fatty acid

Vit $=$ vitamin

$\mathrm{VKR}=$ vitamin $\mathrm{K}$ reductase (NADPH dependent) 


\section{$\underline{\text { AUTHOR QUERY FOR ARS-2011-4437-VER9-BANHEGYI_1P }}$}

AU1: In Ref. 1, please mention the volume number and page range. If these are unavailable, please supply the article's full DOI number. 